\title{
Complex rotation method for the Dirac Hamiltonian
}

\author{
I. A. Ivanov* and Y. K. Ho \\ Institute of Atomic and Molecular Sciences, Academia Sinica, P.O. Box 23-166, Taipei, Taiwan, Republic of China
}

(Received 8 October 2003; published 24 February 2004)

\begin{abstract}
We show that the method of the complex rotation can be applied to the Dirac Hamiltonian. We compute positions and widths of resonance levels of hydrogenlike ions (with $Z=1$ and $Z=10$ ), described relativistically, in the presence of the uniform dc electric field (relativistic Stark effect). We show that, for small $Z$, positions of the resonance levels, given by the relativistic approach, agree well with known nonrelativistic results. The relativistic treatment introduces corrections on the same order both for the resonance positions and widths, and may, therefore, alter widths considerably.
\end{abstract}

DOI: 10.1103/PhysRevA.69.023407

PACS number(s): 32.60. $+\mathrm{i}, 31.30 . J v, 32.30 .-\mathrm{r}$

\section{INTRODUCTION}

The complex rotation method is a well-known tool, allowing to calculate positions and widths of resonances in atomic systems [1-7]. To mention just a few applications, the method has been used in nonrelativistic quantum-mechanical calculations of resonance parameters of doubly excited states $[8,9]$ or Stark resonances [10-12] in such systems as He, $\mathrm{H}^{-}$, or $\mathrm{Ps}^{-}$.

The above mentioned applications of the complex rotation procedure neglected relativistic effects. Recent developments of the experimental technique perfected experimental data to a degree where relativistic effects become observable. For example, in recent publications $[13,14]$ of $2 \ln l^{\prime}$ resonances in helium the authors show that theoretical predictions based on the nonrelativistic approach differ considerably from the experimental data. Only by inclusion of the spin-orbit effects in the scheme of an $R$-matrix multichannel quantum-defect calculation [15] were the experimental results explained.

Another manifestation of the relativistic effects has been reported in Ref. [16], where a peak in the electron- $\mathrm{He}^{+}(1 s)$ scattering has been observed, which is forbidden in the pure $L S$-coupling scheme. The recent experimental studies [17] of the effect of the strong electric field on helium hint at the important role played by the relativistic effects. Also, forbidden in the $L S$ coupling, doubly excited states have been observed in a single-photon excitation from the ground state of helium [18].

These findings indicate that, in the spectra of the doubly excited states, or in the process of the interaction of atomic states with electric field, relativistic effects may play quite an important role. Incorporation of the relativistic effects into the scheme of calculation and, in particular, proper modifications of the complex rotation procedure, allowing to take full account of the relativistic effects, seem to be necessary.

In the present paper we propose a modification of the complex rotation method, taking full account of the relativistic effects. We develop the complex rotation procedure for the Dirac Hamiltonian.

\footnotetext{
*Also at the Institute of Spectroscopy, Russian Academy of Sciences.
}

\section{THEORY}

The Dirac Hamiltonian describing an electron, moving in a central potential $V(r)$, can be written as

$$
\hat{H}_{D}=\boldsymbol{\alpha} \hat{\boldsymbol{p}}+\beta m+V(r)
$$

where $m$ is the rest mass of an electron, $\boldsymbol{\alpha}, \beta$ are $4 \times 4$ matrices. We are not giving their explicit form; it can be found in any standard textbook (e.g., Ref. [19]).

We shall be interested in the case of combination of Coulomb potential and interaction with dc electric field in Eq. (1), i.e., our goal is to describe relativistically Stark effect in hydrogenlike ions.

We shall be employing in the paper two different systems of units. For the purposes of comparison with previous nonrelativistic calculations, we shall use atomic units system. When carrying out the computations based on the Dirac Hamiltonian, relativistic units, in which $\hbar=m=c=1$, where $\hbar, m$, and $c$ are Planck constant, mass of electron, and speed of light, are more convenient.

Using this latter system, the Dirac Hamiltonian describing relativistic Stark effect in hydrogenlike ion with nuclear charge $Z$ can be written as (assuming that dc electric field $F$ is directed along the $z$ axis)

$$
\hat{H}_{D}=\boldsymbol{\alpha} \hat{\boldsymbol{p}}+\beta-\frac{\alpha Z}{r}-\sqrt{\alpha} F z
$$

where $\alpha$ is the fine-structure constant $\left(\alpha^{-1} \approx 137.03599\right)$. Electric-field intensity in Eq. (2) is measured in relativistic units. The conversion of the intensity to more frequently used atomic units of electric-field intensity is given by the relation $F_{\text {rel.units }}=\alpha^{5 / 2} F_{\text {a.u. }}$.

The Hamiltonian (2) acts in the Hilbert space, the elements of which are four-component functions (bispinors) [19]:

$$
\Psi=\left(\begin{array}{l}
\Phi \\
\Xi
\end{array}\right),
$$

where $\Phi, \Xi$ are the usual two-component spinors. The scalar product of elements $\Psi_{1}, \Psi_{2}$ of this Hilbert space is defined as follows [19]: 


$$
\left\langle\Psi_{1}, \Psi_{2}\right\rangle=\left(\Phi_{1}, \Phi_{2}\right)+\left(\Xi_{1}, \Xi_{2}\right) .
$$

Formally, the complex rotation method can be described [4] as the complex transformation of the spatial variables: $\boldsymbol{r}$ $\rightarrow \boldsymbol{r} \exp (i \theta)$, where $\theta$ is the so-called rotation angle. Under this transformation the Dirac Hamiltonian (2) assumes the form

$$
\hat{H}_{D}(\theta)=\left(\boldsymbol{\alpha} \hat{\boldsymbol{p}}-\frac{\alpha Z}{r}\right) \exp (-i \theta)+\beta-\sqrt{\alpha} F z \exp (i \theta) .
$$

Marked difference of this expression, compared to the nonrelativistic complex rotated Hamiltonian, is the absence of terms scaling under complex rotation as $\exp (-2 i \theta)$ (terms representing kinetic energy in the nonrelativistic Hamiltonian). In the case of the Dirac Hamiltonian both cynetic and Coulomb potential parts of the Hamiltonian scale as $\exp (-i \theta)$. In addition, a term with $\beta$ matrix appears, which does not scale at all.

To proceed further, we must restrict Hamiltonian (5) to subspaces corresponding to states of given symmetry (total momentum $J$ and parity $P$ ).

Such states are given by the following four-component functions [19]:

$$
\Psi_{P J}^{M}=\frac{1}{r}\left(\begin{array}{c}
f(r) Y_{l J}^{M}(\boldsymbol{n}, \sigma) \\
g(r) Y_{l^{\prime} J}^{M}(\boldsymbol{n}, \sigma)
\end{array}\right)
$$

where $Y_{l J}^{M}(\boldsymbol{n}, \sigma)$ and $Y_{l^{\prime} J}^{M}(\boldsymbol{n}, \sigma)$ are the two-component spinors obtained in a usual way by coupling orbital momentum $l\left(l^{\prime}\right)$ and the spin momentum $1 / 2$ to produce a state with a given total $J$ and its projection $M_{J}=M$. Parity of the state defined by Eq. (6) is determined by $l$; it is equal to $(-1)^{l}$. For a given $J$ and $l$ in Eq. (6) $l^{\prime}$ may assume values $l^{\prime}=l \pm 1$ (of course, only one of these possibilities is realized for a given $J)$. Functions $f(r), g(r)$ are arbitrary functions of $r$.

Hamiltonian (5) acting on the functions of the type (6) with given $J$ yields [19]

$$
\begin{aligned}
\hat{H}_{D}(\theta) \Psi_{P J}^{M}= & \alpha_{r}\left(\hat{p}_{r}-\frac{i \omega\left(J+\frac{1}{2}\right)}{r} \beta\right) \exp (-i \theta) \Psi_{P J}^{M} \\
& +\left(\beta-\frac{\alpha Z}{r} \exp (-i \theta)-\sqrt{\alpha} F z \exp (i \theta)\right) \Psi_{P J}^{M},
\end{aligned}
$$

where

$$
\begin{aligned}
& \alpha_{r}=\left(\begin{array}{cc}
0 & \boldsymbol{\sigma n} \\
\boldsymbol{\sigma n} & 0
\end{array}\right), \\
& \beta=\left(\begin{array}{cc}
1 & 0 \\
0 & -1
\end{array}\right),
\end{aligned}
$$

and $\hat{p}_{r}=-i(1 / r)(d / d r) r, \quad \boldsymbol{n}=\boldsymbol{r} / r$ a unit vector along $\boldsymbol{r}$, $\boldsymbol{\sigma}$-Pauli matrices. The parameter $\omega$ in Eq. (7) is defined as follows. If $l=J-\frac{1}{2}, \omega=-1$, if $l=J+\frac{1}{2}, \omega=1$.

To diagonalize the complex rotated Hamiltonian (7), one should choose a proper basis in each of the subspaces (6). A convenient basis is provided by the following set:

$$
\Psi_{P J}^{a, M}=\frac{1}{r}\left(\begin{array}{c}
F(r) Y_{l J}^{M}(\boldsymbol{n}, \sigma) \\
0
\end{array}\right)
$$

and

$$
\Psi_{P J}^{b, M}=\frac{1}{r}\left(\begin{array}{c}
0 \\
i G(r) Y_{l^{\prime} J}^{M}(\boldsymbol{n}, \sigma)
\end{array}\right),
$$

where each of the functions $F(r)$ and $G(r)$ is a Slater-type orbital:

$$
\begin{aligned}
& F(r)=r^{i} e^{-\mu_{i} r}, \\
& G(r)=r^{j} e^{-\nu_{j} r},
\end{aligned}
$$

where $i=l+1, l+2, \ldots, j=l^{\prime}+1, l^{\prime}+2, \ldots$.

The Hamiltonian (7) has nonzero matrix elements only between states with the same $M_{J}$ (it is the only conserved quantity in the problem). The Hamiltonian matrix, therefore, is to be diagonalized in the subspace spanned by functions (11), corresponding to a given $M_{J}$. Below we present numerical results and details of the numerical procedure for the states of hydrogenlike ions.

\section{NUMERICAL RESULTS}

We consider only the states with $M_{J}=1 / 2$ in the present paper. The matrix of the Hamiltonian (7) was computed in the basis, constructed from the functions (11) and (12), as follows.

For a given total momentum $J$ all possible values of $l, l^{\prime}$ in Eq. (11), compatible with these $J$ and parity (there are only finite number of them), participated in the building up of the energy matrix. For each $l$ we constructed 24 basis Slater functions $F(r)$ of the type (12), where parameter $i$ assumed values $l+1, l+2, l+3$, and parameter $\mu_{i}$ assumed values $\alpha Z, 2 \alpha Z, 4 \alpha Z, 8 \alpha Z, 16 \alpha Z, 32 \alpha Z, 64 \alpha Z$. Radial functions $G(r)$ were constructed quite analogously with only difference that parameter $j$ assumed values $l^{\prime}+1, l^{\prime}+2, l^{\prime}$ +3 , and parameter $\nu_{j}$ assumed values from the same set as the parameter $\mu_{i}$ above. Such choice of the parameters was motivated by the fact that Hamiltonian matrix, calculated in the basis of the Slater orbitals, becomes ill conditioned rapidly, if only orbitals with the same $\mu_{i}$ (or $\nu_{j}$ ) participate in the calculation of the energy matrix. To make the calculation free of these numerical problems, the parameters of the exponential functions should assume values spread over rather large interval, which makes overlaps of these functions small.

The basis functions with both parities and total momenta 


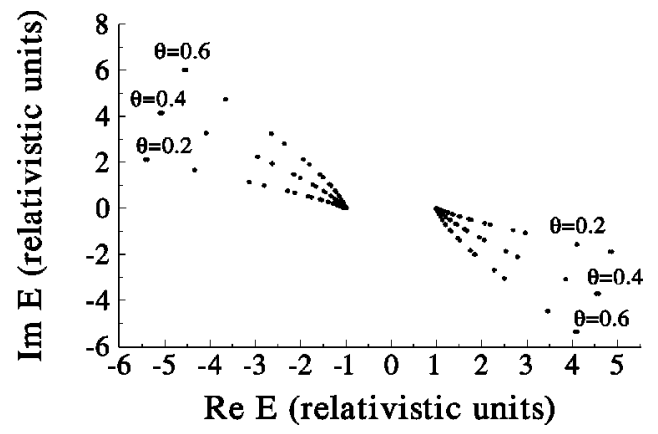

FIG. 1. Spectrum of the rotated Dirac Hamiltonian for different values of $\theta$.

$J$ assuming values $J=\frac{1}{2}, \ldots, \frac{15}{2}$ were included in the calculation of the energy matrix.

With the basis set chosen as described above, we computed the matrix $H_{i j}(\theta)$ of the Hamiltonian (7). Since the basis chosen is not an orthonormal one, we need an overlap matrix $R_{i j}$ as well. Thus, solving the Schrödinger equation for the complex rotated Dirac Hamiltonian (7) amounted to the solution of the generalized eigenvalue problem:

$$
\operatorname{det}[H(\theta)-E R]=0
$$

The diagonalization of this problem produces complex eigenvalues. With the basis chosen as above, the dimension of all the matrices involved was $N=896$.

From the theory of the complex rotation method in the nonrelativistic quantum mechanics, it is known [4] that, as a result of the complex rotation transformation, the continuous spectrum of the Hamiltonian rotates clockwise (where angle of rotation is $2 \theta$ ). In terms of the complex eigenvalues of nonrelativistic analog of the complex eigenvalue problem (13), this property can be restated as follows [4]. Some eigenvalues of the complex eigenvalue problem represent the "rotated" continuum spectrum, and they are aligned along the corresponding straight lines in the complex plane; some of the eigenvalues, not lying on these lines, represent resonances or bound states.

In Fig. 1 we present eigenvalues of the complex eigenvalue problem (13) for the Dirac Hamiltonian (7) in the field-free case $(F=0)$. The basis set described above was used.

From Fig. 1 one can see that, as far as its action on the spectrum is concerned, the relativistic version of the complex rotation procedure possesses the same property as in the nonrelativistic case, with one important difference. The Dirac Hamiltonian is unbounded from below, and continuous spectrum for this operator is a union of two intervals of the real axis: $(-\infty,-1)$ and $(1,+\infty)$ (we remind that we are using relativistic units where mass of electron is 1). In Fig. 1 we represent eigenvalues of the problem (13) for three different values of $\theta: 0.2,0.4,0.6$ (nuclear charge $Z=10$ ). One can see from Fig. 1 that the spectrum of the rotated Hamiltonian (7) rotates clockwise, the angle between the tangent line to the curve representing continuous spectrum and the real axis at the points $-1,1$ being approximately $2 \theta$, in accordance

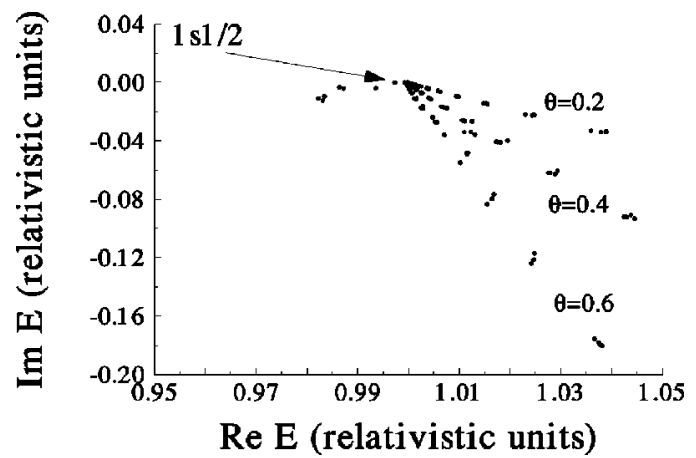

FIG. 2. Part of the spectrum of the rotated Dirac Hamiltonian near the point $E=1$ (rel.units) for different values of $\theta$.

with what one would expect from the relativistic expression $\pm \sqrt{1+k^{2} \exp (-2 i \theta)}$ for small $k$.

To see better how the bound state $1 s_{1 / 2}$ exhibits itself, in Fig. 2 we present, under magnification, the part of the spectrum of the rotated Hamiltonian of the previous example. As in nonrelativistic case, the eigenvalues of Eq. (13) representing bound states (the state $1 s_{1 / 2}$ is pointed by the arrow in the figure), or resonance states, do not change with $\theta$, while eigenvalues representing continuous spectrum rotate. The former are associated with resonance complex energies $E$ $=E_{r}-i(\Gamma / 2)$, where $E_{r}$ is resonance position and $\Gamma$ its width.

In the tables presented below we use atomic units more frequently used in atomic physics. To facilitate comparison of energies, we also subtract the rest mass energy from our results for the real part of the energy, the conversion of the complex energies, therefore, being given by $E$ (a.u.) $=\alpha^{-2}[E$ (rel.units $\left.)-1\right]$.

In the field-free case the calculation described above yields for the ground level of hydrogen $E=-0.50000666$ a.u., to be compared to -0.50000665 a.u., the value predicted by the Sommerfeld formula. For the $N=2$ states of the hydrogenlike ion with $Z=10$, the field-free results obtained with the help of the basis described above are as follows: $\quad 2 s_{1 / 2}, \quad E=-12.5208746$ a.u.; $2 p_{1 / 2}, \quad E$ $=-12.5208596$ a.u.; $2 p_{3 / 2}, E=-12.5041630$ a.u.; to be compared with the energies given by the Sommerfeld formula: $2 s_{1 / 2}, 2 p_{1 / 2}: E=-12.52085966$ a.u.; and $2 p_{3 / 2}: E$ $=-12.50416303$ a.u. This suggests that the relative numerical uncertainty of the present calculation is on the order of $10^{-7}$.

Due to the fact that the basis used is finite, the complex eigenvalues vary slowly with $\theta$. To investigate this $\theta$ dependence, we performed repeated diagonalizations of the eigenvalue problem (13) with different $\theta$ values. The results are presented in Table I.

As usual, the value for which this variation is minimal is chosen as an optimal value (in our case it is $\theta \approx 0.50$ ), and the calculation for different fields is performed with this value of the rotation angle $\theta$. It is with the value so chosen that the results for the ground level of hydrogen presented below have been obtained. One further remark concerning the data from Table I should be made. The magnitude of the dispersion of the complex energies around the value obtained 
TABLE I. Position and width of the ground level of hydrogen given by complex rotation of the Dirac Hamiltonian for different $\theta$ values ( $F=0.1$ a.u.).

\begin{tabular}{ccc}
\hline \hline$\theta$ & $\operatorname{Re} E$ (a.u.) & $\Gamma$ (a.u.) \\
\hline 0.10 & -0.5274457378 & 0.014832887 \\
0.20 & -0.5274269403 & 0.014545690 \\
0.30 & -0.5274238506 & 0.014536681 \\
0.40 & -0.5274235931 & 0.014536178 \\
0.50 & -0.5274235418 & 0.014536226 \\
0.60 & -0.5274235047 & 0.014536292 \\
0.70 & -0.5274233608 & 0.014536470 \\
\hline \hline
\end{tabular}

for the optimal value of $\theta, \theta=0.50$, can be used as an estimation of the numerical uncertainty of our calculation. It can be seen from the data in the table that this uncertainty can be estimated as being on the order of $10^{-7}$ a.u. both for the positions and widths.

The stability of the results with respect to the variations of the size of the basis has been also examined. In Table II we present the results of the several calculations of position and width of the $1 s_{1 / 2}$ resonance in hydrogen for $F=0.1$ a.u., when the basis described above is modified. We examined convergence of our results with respect to the maximum value of the angular momentum $J$. In the table we show the results obtained when this maximum $J$ value was $\frac{9}{2}, \frac{11}{2}, \frac{13}{2}$, and $\frac{15}{2}$.

As can be seen, the results exhibit good convergence, the difference between calculations with $J_{\max }=\frac{13}{2}$ and $J_{\max }=\frac{15}{2}$ being on the order of $10^{-7}$ a.u., in agreement with our previous estimation of the numerical uncertainty.

As a final consistency check, we studied the results which our approach gives in the nonrelativistic limit. To do that we repeated the calculation with $J_{\max }=\frac{15}{2}$ with the "modified" value of the fine-structure constant, i.e., in the Hamiltonian, the fine-structure constant was ascribed a value 100 times less than its actual value. Since such diminishing of the finestructure constant corresponds to the 100-fold increase of the value of the speed of light, such calculation must closely reproduce results given by the purely nonrelativistic approach. That this is indeed so can be seen from the data presented in Table II. The results of the nonrelativistic calculation [20] and the present calculation based on the Dirac

TABLE II. Results of the different calculations for the ground level of hydrogen $(F=0.1$ a.u. $)$.

\begin{tabular}{lccc}
\hline \hline$J_{\max }$ & Basis size & $\operatorname{Re} E$ (a.u.) & $\Gamma$ (a.u.) \\
\hline$\frac{9}{2}$ & 560 & -0.5274153234 & 0.014500308 \\
$\frac{11}{2}$ & 672 & -0.5274275909 & 0.014539062 \\
$\frac{13}{2}$ & 784 & -0.5274226229 & 0.014537540 \\
$\frac{15}{2}$ & 896 & -0.5274235418 & 0.014536226 \\
$\frac{15}{2}$ & 896 & -0.5274182645 & 0.014537912 \\
& & $-0.52741818^{\mathrm{a}}$ & 0.014538 \\
\hline \hline
\end{tabular}

${ }^{\mathrm{a}}$ Reference [20].
Hamiltonian, the value of the fine-structure constant modified as noted above, agree quite well.

For the calculation of $N=2$ states of hydrogenlike ion with $Z=10$, presented below, the numerical uncertainty, estimated quite analogously, was found to be on the order of $10^{-5}$ a.u. for real and imaginary parts of energy. We do not dwell on the details of the calculation for $Z=10$; it has been performed in essentially the same way as that for $Z=1$.

In Table III we present the results of the present calculation for the positions and widths of the ground level of hydrogen and $N=2$ levels of the hydrogenlike ion with $Z$ $=10$ (all levels having $M_{J}=\frac{1}{2}$ ) as functions of the intensity of the dc electric field.

The results are compared to the nonrelativistic results. For some of the data (for $Z=1$ ) we used the results available in the literature [20-22]; for $Z=10$ we performed a separate nonrelativistic calculation, applying the complex rotation procedure to the nonrelativistic Hamiltonian. The technique of the nonrelativistic complex rotation calculations is well known [4], and we shall not present the details here. It should be noted, perhaps, that the three $N=2, M_{J}=\frac{1}{2}$ Stark levels, presented in the table, correspond, in the nonrelativistic limit, to the following three levels of the nonrelativistic $N=2$ Stark effect: $2 s_{1 / 2}, 2 p_{1 / 2}$ correspond to the levels $2 s$, $2 p$ with $M_{L}=0$ (where $M_{L}$ is orbital momentum projection), $2 p_{3 / 2}$ corresponds to the level $2 p$ with $M_{L}=1$.

We remind that, both for $Z=1$ and $Z=10$ data, the relative uncertainty was found to be on the order of $10^{-7}$, implying that, for the sufficiently large field values ( $F$ $\geqslant 0.04$ a.u. for $Z=1$ and $F \geqslant 4$ a.u. for $Z=10$ ), the relativistic effects upon the widths can be reliably distinguished from the mere numerical uncertainty of the calculation.

As one can see from Table III, for $Z=1$ the relativistic treatment introduces only a very minor correction. This, of course, should be expected, since, in the leading order, the relativistic correction is on the order of $\alpha^{2} Z^{4}$, ( $\alpha$-the finestructure constant), which leads to correction on the order of $10^{-6}$ a.u. for hydrogen. For $Z=10$ the relativistic corrections play more important role, and are, as can be seen from the data presented in the table, on the order of $10^{-3}-10^{-2}$ a.u. One should note that the relativistic effects for positions and widths have about the same order of magnitude, i.e., relatively to their nonrelativistic values, the positions and widths are changed by roughly the same absolute amount. For example, as follows from the data in Table III, for the ground state of hydrogen, for $F=0.04$ a.u., the account of the relativistic effects shifts position of the Stark resonance by the amount of $6 \times 10^{-6}$ a.u., and its width by the amount of 2 $\times 10^{-7}$ a.u. It means that, while for the position of the resonance the relativistic effects amount to a negligible fraction of $1 \%$, for the width, the relativistic effects manifest themselves at the level of $1 \%$. Even more dramatic example could be found, perhaps, if we considered smaller field values, for which the widths are smaller. For such fields $(F \sim 0.03$ a.u. and less for hydrogen) we could not carry out numerically reliable calculations of the widths, since the widths become less than the estimated numerical uncertainty. Still, we believe, one may conclude from the data presented in Table III that the account of the relativistic effects may modify widths 
TABLE III. Positions and widths of the $1 s_{1 / 2}$ level of hydrogen $(Z=1)$, and the levels $2 s_{1 / 2}, 2 p_{1 / 2}, 2 p_{3 / 2}\left(M_{J}=1 / 2, Z=10\right)$, as functions of electric-field intensity, complex rotated Dirac Hamiltonian (CRD), and nonrelativistic results.

\begin{tabular}{|c|c|c|c|c|}
\hline \multirow[b]{2}{*}{$F$ (a.u.) } & \multicolumn{2}{|c|}{ CRD } & \multicolumn{2}{|c|}{ Nonrelativistic results } \\
\hline & $\operatorname{Re} E$ (a.u.) & $\Gamma$ (a.u.) & $\operatorname{Re} E$ (a.u.) & $\Gamma$ (a.u.) \\
\hline \multicolumn{5}{|c|}{$1 s_{1 / 2} Z=1$} \\
\hline 0 & -0.50000666 & 0 & -0.5 & 0 \\
\hline 0.01 & -0.50023223 & 0 & & \\
\hline 0.02 & -0.50091585 & 0 & & \\
\hline 0.03 & -0.50208083 & 0 & -0.50207427 & \\
\hline 0.04 & -0.50377804 & $0.41 \times 10^{-5}$ & -0.50377159 & $0.39 \times 10^{-5}$ \\
\hline 0.05 & -0.50611173 & $0.774 \times 10^{-4}$ & -0.50610543 & $0.772 \times 10^{-4}$ \\
\hline 0.06 & -0.50920955 & $0.5152 \times 10^{-3}$ & -0.50920345 & $0.5151 \times 10^{-3}$ \\
\hline 0.07 & -0.51308260 & $0.18473 \times 10^{-2}$ & -0.51307677 & $0.18474 \times 10^{-2}$ \\
\hline 0.08 & -0.51756619 & $0.45391 \times 10^{-2}$ & -0.51756062 & $0.45397 \times 10^{-2}$ \\
\hline 0.09 & -0.52241821 & $0.87828 \times 10^{-2}$ & -0.52241281 & $0.87840 \times 10^{-2}$ \\
\hline 0.1 & -0.52742354 & $0.145362 \times 10^{-1}$ & -0.52741818 & $0.145381 \times 10^{-1}$ \\
\hline \multicolumn{5}{|c|}{$2 s_{1 / 2} Z=10$} \\
\hline 0 & -12.52086 & 0 & -12.5 & 0 \\
\hline 2 & -13.14961 & $0.2 \times 10^{-4}$ & -13.13533 & $0.1 \times 10^{-4}$ \\
\hline 4 & -13.86772 & $0.44 \times 10^{-3}$ & -13.85488 & $0.45 \times 10^{-3}$ \\
\hline 6 & -14.71966 & $0.6554 \times 10^{-1}$ & -14.70918 & $0.6676 \times 10^{-1}$ \\
\hline 8 & -15.67420 & 0.42773 & -15.66554 & 0.43223 \\
\hline 10 & -16.61683 & $0.108039 \times 10^{1}$ & -16.60929 & $0.108833 \times 10^{1}$ \\
\hline \multicolumn{5}{|c|}{$2 p_{1 / 2} Z=10$} \\
\hline 0 & -12.52086 & 0 & -12.5 & 0 \\
\hline 2 & -11.94869 & $0.2 \times 10^{-4}$ & -11.93274 & $0.1 \times 10^{-4}$ \\
\hline 4 & -11.44692 & $0.3 \times 10^{-4}$ & -11.43054 & $0.2 \times 10^{-4}$ \\
\hline 6 & -11.01991 & $0.593 \times 10^{-2}$ & -11.00366 & $0.609 \times 10^{-2}$ \\
\hline 8 & -10.68390 & $0.8444 \times 10^{-1}$ & -10.66829 & $0.8619 \times 10^{-1}$ \\
\hline 10 & -10.40443 & 0.32374 & -10.38915 & 0.32853 \\
\hline \multicolumn{5}{|c|}{$2 p_{3 / 2} Z=10$} \\
\hline 0 & -12.50416 & 0 & -12.5 & 0 \\
\hline 2 & -12.54670 & 0 & -12.53157 & 0 \\
\hline 4 & -12.64625 & $0.8 \times 10^{-4}$ & -12.63169 & $0.8 \times 10^{-4}$ \\
\hline 6 & -12.84044 & $0.2126 \times 10^{-1}$ & -12.82715 & $0.2178 \times 10^{-1}$ \\
\hline 8 & -13.13801 & 0.20423 & -13.12615 & 0.20731 \\
\hline 10 & -13.46349 & 0.62140 & -13.45247 & 0.62792 \\
\hline
\end{tabular}

considerably (at the level of a few percent) even for such light system as hydrogen, when fields are small.

It is worthwhile to compare the present results, given by the nonperturbative complex rotated Dirac Hamiltonian (CRD) procedure and the relativistic perturbation results, obtained if the term describing interaction with the dc electric field in the Hamiltonian (2) is considered as a perturbation.

For the energy of the ground state of hydrogen the standard prescriptions of the Rayleigh-Schrödinger perturbation theory lead to the expansion

$$
E(F) \sim E_{0}+E_{1} F^{2}+\cdots .
$$

The coefficient $E_{0}$ is the field-free energy of the ground state of the hydrogen atom given by the Sommerfeld formula; coefficient $E_{1}$ can be computed analytically [23,24], and its value is $E_{1} \approx-2.24987574$. In Table IV we compare the results produced by the CRD procedure, and results,
TABLE IV. Position of the $1 s_{1 / 2}$ level of hydrogen $(Z=1)$ as function of applied dc field, CRD, and relativistic perturbative results.

\begin{tabular}{lcc}
\hline \hline$F($ a.u. $)$ & CRD & Perturbation theory \\
\hline 0 & -0.50000666 & -0.50000666 \\
0.01 & -0.50023223 & -0.50023164 \\
0.02 & -0.50091585 & -0.50090661 \\
0.03 & -0.50208083 & -0.50203154 \\
0.04 & -0.50377804 & -0.50360646 \\
0.05 & -0.50611173 & -0.50563135 \\
0.06 & -0.50920955 & -0.50810621 \\
0.07 & -0.51308260 & -0.51103105 \\
0.08 & -0.51756619 & -0.51440586 \\
0.09 & -0.52241821 & -0.51823065 \\
0.1 & -0.52742354 & -0.52250541 \\
\hline \hline
\end{tabular}


which the perturbation series (14), with the first two terms retained, yields for different values of the field strength. Only the results for the position of the $1 s_{2 / 2}$ resonance are presented in the table. From the coefficients of the perturbation series, it is possible to extract information about the widths as well [22], but to do that, one should know a fairly large number of the perturbation coefficients.

The data in Table IV show that the perturbation expansion produces quite accurate results for the position of the $1 s_{1 / 2}$ resonance in hydrogen for not very large field strengths ( $F$ $\sim 0.03$ a.u., or less). With increasing field strength, the accuracy of the perturbation expansion deteriorates rapidly.

\section{CONCLUSION}

To conclude, we proposed a modification of the standard procedure of the complex rotation method, allowing to include relativistic effects to a full extent. We have shown that the complex rotation transformation can be applied to the Dirac Hamiltonian (CRD procedure).

As we saw from the previous discussion, the relativistic treatment introduces relatively minor corrections to the resonance positions, while there may be situations when relativistic effects for widths may be quite significant even for hydrogen. This shows the importance and necessity of relativistic treatment.

The present approach remains valid as long as the Dirac equation based on the Dirac Hamiltonian (2) provides an adequate tool for the description of an electron in the static external fields. It means, of course, that we must remain within the domain of the field strengths, where one can neglect the multiparticle character of the QED. In particular, the external dc electric field must be much less than the critical value $E_{\text {crit }} \sim \alpha^{-3}$ a.u. $\left(\sim 10^{16} \mathrm{~V} / \mathrm{cm}\right)$ for which the multiparticle processes, such as electron-positron pair creation, become significant.

As for generalizing the CRD to the systems with more than one electron, one should keep in mind that, strictly speaking, there is no Dirac equation for the system with two electrons in quantum electrodynamics. One may, however, adopt less rigorous and more practical approach, and attempt to describe the relativistic two-electron system by means of a model relativistic Hamiltonian (e.g., in the spirit of the Dirac-Fock approach). Then, the straightforward application of the present procedure meets no essential obstacles, apart from some difficulties of technical character due to increased complexity of matrix elements. One should not forget, of course, that such description is not a truly ab initio approach; it might be considered as a tool, allowing to include the leading relativistic corrections in the theory of atomic resonances.

\section{ACKNOWLEDGMENT}

This work was supported by the National Science Council of ROC.
[1] E. Balslev and J.M. Combs, Commun. Math. Phys. 22, 296 (1971).

[2] B. Simon, Commun. Math. Phys. 27, 1 (1972).

[3] C. Cerjan, R. Hedges, C. Holt, W.P. Reinhardt, K. Scheibner, and J.J. Wendoloski, Int. J. Quantum Chem. 14, 393 (1978).

[4] Y.K. Ho, Phys. Rep. 99, 1 (1983).

[5] W.P. Reinhardt, Annu. Rev. Phys. Chem. 33, 223 (1982).

[6] B.R. Junker, Adv. At. Mol. Phys. 18, 208 (1982).

[7] A. Maquet, Shih-I. Chu, and W.P. Reinhardt, Phys. Rev. A 27, 2946 (1983).

[8] Y.K. Ho, Phys. Rev. A 44, 4154 (1991).

[9] I.A. Ivanov and Y.K. Ho, Phys. Rev. A 61, 032501 (2000).

[10] Y.K. Ho, Z. Phys. D: At. Mol. Clusters 38, 191 (1996).

[11] Y.K. Ho and J. Callaway, Phys. Rev. A 50, 4941 (1994).

[12] Y.K. Ho and I.A. Ivanov, Phys. Rev. A 63, 062503 (2001).

[13] J.E. Rubensson, C. Sathe, S. Cramm, B. Kessler, S. Stranges, R. Richter, M. Alagia, and M. Coreno, Phys. Rev. Lett. 83, 947 (1999).

[14] K.C. Prince et al., J. Synchrotron Radiat. 5, 565 (1998).
[15] T.W. Gorczyca, J.-E. Rubensson, C. Sathe, M. Strom, M. Agaker, D. Ding, S. Stranges, R. Richter, and M. Alagia, Phys. Rev. Lett. 85, 1202 (2000).

[16] A.A. Wils, E. Sokell, T.W. Gorczyca, X. Feng, M. Wiedenhoeft, S.E. Kanton, and N. Berrah, J. Phys. B: At. Mol. Opt. Phys. 35, L367 (2002).

[17] J.R. Harries, J.P. Sullivan, J.B. Sternberg, S. Obara, T. Suzuki, P. Hammond, J. Bozek, N. Berrah, M. Halka, and Y. Azuma, Phys. Rev. Lett. 90, 133002 (2003).

[18] F. Penent, P. Lablanquie, R.I. Hall, M. Zitnik, K. Bucar, S. Stranges, R. Richter, M. Alagia, P. Hammond, and J.G. Lambourne, Phys. Rev. Lett. 86, 2758 (2001).

[19] A. Messiah, Quantum Mechanics (Wiley, New York, 1976).

[20] L. Benassi and V. Grecchi, J. Phys. B 13, 911 (1980).

[21] I.W. Herbst and B. Simon, Phys. Rev. Lett. 41, 67 (1978).

[22] I.A. Ivanov, Phys. Rev. A 56, 202 (1997).

[23] R. Szmytkowski, J. Phys. B: At. Mol. Opt. Phys. 30, 825 (1997).

[24] R. Szmytkowski, Phys. Rev. A 65, 012503 (2001). 